\title{
Spherical Wave Propagation in a Nonlinear Elastic Medium
}

\author{
Valeri Korneev \\ Earth Sciences Division \\ Lawrence Berkeley National Laboratory
}

August 19, 2009

\begin{abstract}
Nonlinear propagation of spherical waves generated by a point-pressure source is considered for the cases of monochromatic and impulse primary waveforms. The nonlinear five-constant elastic theory advanced by Murnaghan is used where general equations of motion are put in the form of vector operators, which are independent of the coordinate system choice. The ratio of the nonlinear field component to the primary wave in the far field is proportional to $\ln (r)$ where $r$ is a propagation distance. Near-field components of the primary field do not contribute to the far field of nonlinear component.
\end{abstract}

\section{Introduction}

This work was done in 1993 during a multi-lab DOE project on nonlinear seismic effects. The author's contribution back then resulted in two reports with theoretical results (Korneev et al., 1998; Korneev, 1998). The experimental LBNL results are covered in Daley et al. (1992). By the end of the project, the results for a point-pressure source had neither a quantitative part nor data and were abandoned until now. Currently, there is a renewed interest to nonlinear phenomenon in seismic prospecting. Some publications demonstrate correlations between the observed nonlinear properties of seismic fields and hydrocarbon reservoir characteristics (Zhukov et al.,2008). In this connection, the results of the report might be of interest for geophysicists dealing with seismic nonlinear phenomenon. Another possible application of the considered problem is the physics of powerful underground explosions. The results of this report take into account the elastic nonlinearity within a framework of the five-constant Murnaghan

${ }^{0}$ Earth Sciences Division, Lawrence Berkeley National Laboratory, Berkeley, California, 94720, USA 
theory (Murnaghan, 1951), and the relative smallness of the nonlinear field component compared to the linear one. The author assumes that the basic equations and expressions of this theory can be found in several studies (Zarembo and Krasil'nikov, 1971,1966; Taylor and Rollins, 1999; Landau and Lifshitz, 1953;Hughes and Kelly, 1953; Gedroits and Krasil'nikov, 1963) and the other sited publications.

\section{Equations of motion}

Following the work of Jones and Kobett (1963), we will use the equation of motion in an perfectly elastic solid

$$
\rho \frac{\partial^{2} U_{i}}{\partial t^{2}}-\mu \frac{\partial^{2} u_{i}}{\partial x_{k}^{2}}-(\lambda+\mu) \frac{\partial^{2} u_{k}}{\partial x_{k} \partial x_{i}}=F_{i}
$$

where $F_{i}$, the $i^{i t h}$ component of $\mathbf{F}$, has a value of the second order in smallness and is given by:

$$
\begin{gathered}
F_{i}=C_{1}\left(\frac{\partial^{2} u_{s}}{\partial x_{k}^{2}} \frac{\partial u_{s}}{\partial x_{i}}+\frac{\partial^{2} u_{s}}{\partial x_{k}^{2}} \frac{\partial u_{i}}{\partial x_{s}}+2 \frac{\partial^{2} u_{i}}{\partial x_{s} \partial x_{k}} \frac{\partial u_{s}}{\partial x_{k}}\right)+C_{2}\left(\frac{\partial^{2} u_{s}}{\partial x_{i} \partial x_{k}} \frac{\partial u_{s}}{\partial x_{k}}+\frac{\partial^{2} u_{k}}{\partial x_{s} \partial x_{k}} \frac{\partial u_{i}}{\partial x_{s}}\right) \\
+C_{3} \frac{\partial^{2} u_{i}}{\partial x_{k}^{2}} \frac{\partial u_{s}}{\partial x_{s}}+C_{4}\left(\frac{\partial^{2} u_{k}}{\partial x_{s} \partial x_{k}} \frac{\partial u_{s}}{\partial x_{i}}+\frac{\partial^{2} u_{s}}{\partial x_{i} \partial x_{k}} \frac{\partial u_{k}}{\partial x_{s}}\right)+C_{5} \frac{\partial^{2} u_{k}}{\partial x_{i} \partial x_{k}} \frac{\partial u_{s}}{\partial x_{s}}
\end{gathered}
$$

where constants

$$
\begin{gathered}
C_{1}=\mu+\frac{A}{4}, \quad C_{2}=\lambda+\mu+\frac{A}{4}+B, \quad C_{3}=\lambda+B \\
C_{4}=\frac{A}{4}+B, \quad C_{5}=B+2 C
\end{gathered}
$$

contain the nonlinear constants $A, B, C$ by Landau and Lifshitz (1953), which can be expressed through the constants introduced by Murnaghan (1951).

After some algebra, the nonlinear term (2) can be represented in the general form:

$$
\mathbf{F}=C_{1} \mathbf{W}_{1}+C_{2} \mathbf{W}_{2}+C_{3} \mathbf{W}_{3}+C_{4} \mathbf{W}_{4}+C_{5} \mathbf{W}_{5}
$$

where

$$
\mathbf{W}_{1}=[\Delta \mathbf{u} \times \operatorname{rot} \mathbf{u}]+\frac{1}{2} \nabla \Delta(\mathbf{u u})+\operatorname{div} \mathbf{u} \Delta \mathbf{u}-\Delta[u \times \operatorname{rot} \mathbf{u}]+\operatorname{rot}[\mathbf{u} \times \Delta u]-\mathbf{u} \Delta \operatorname{div} \mathbf{u}
$$




$$
\begin{gathered}
\mathbf{W}_{2}=\frac{1}{2}(\mathbf{W}+\nabla(\operatorname{rotrot} \mathbf{u} \mathbf{u})-\operatorname{rot}[\nabla \operatorname{div} \mathbf{u} \times \mathbf{u}]-[\nabla \operatorname{div} \mathbf{u} \times \operatorname{rot} \mathbf{u}]) \\
\mathbf{W}_{3}=\operatorname{div} \mathbf{u} \Delta \mathbf{u} \\
\mathbf{W}_{4}=\frac{1}{2}(\mathbf{W}+[\nabla \operatorname{div} \mathbf{u} \times \operatorname{rot} \mathbf{u}]-\operatorname{rot}[\nabla \operatorname{div} \mathbf{u} \times \mathbf{u}]-\nabla \operatorname{div}[\mathbf{u} t i m e s r o t \mathbf{u}]) \\
\mathbf{W}_{5}=\operatorname{div} \mathbf{u} \nabla \operatorname{div} \mathbf{u} \\
\mathbf{W}=\frac{1}{2} \nabla \Delta(\mathbf{u u})-\mathbf{u} \Delta \operatorname{div} \mathbf{u}+\operatorname{div} \mathbf{u} \nabla \operatorname{div} \mathbf{u}
\end{gathered}
$$

independent of a choice of coordinate system.

\section{Spherical Symmetry}

In the case of a full spherical symmetry, we have radial displacement only, and the equation of motion (1) can be reduced to a scalar form

$$
\begin{gathered}
\frac{d^{2} u}{d r^{2}}+\frac{2}{r} \frac{d u}{d r}-\frac{2}{r^{2}} u-\frac{1}{v^{2}} \frac{d^{2} u}{d t^{2}}=-\frac{F}{\lambda+2 \mu}, \quad v=\sqrt{\frac{\lambda+2 \mu}{\rho}} \\
F=D\left(\frac{d u}{d r}+\frac{2}{r} u\right)\left(\frac{d^{2} u}{d r^{2}}+\frac{2}{r} \frac{d u}{d r}-\frac{2}{r^{2}} u\right) \\
+E\left(\frac{d^{2} u}{d r^{2}} \frac{d u}{d r}+\frac{1}{r}\left(\frac{d u}{d r}\right)^{2}-\frac{u^{2}}{r^{3}}\right)
\end{gathered}
$$

where we use the notations

$$
\begin{gathered}
D=\lambda+2 B+2 C=\lambda+2 l \\
E=2(\lambda+3 \mu+A+2 B)=2(\lambda+3 \mu+2 m)
\end{gathered}
$$


with nonlinear constants $l$ and $m$ by Murnaghan (1951). The similar equation was obtained in Beresnev (1990) by direct transformation of equation (2) where the numerical finite difference scheme was used to solve it. Here we develop an analytical approach using the relative smallness of $F$, which allows one to seek the solution in the form

$$
u=u_{0}+u_{1}
$$

where $u_{0}$ is the solution of reduced equation (12) when $F$ is assumed equal to zero. Putting (16) into (12) and assuming that $\left|u_{0}\right|>\left|u_{1}\right|$ we obtain a linear equation for $u_{1}$, where the left-hand side contains components of $u_{1}$ and the right-hand side $F$ depends exclusively on the previously determined function $u_{0}$. We also can take an advantage of $u_{0}$ being the solution of the reduced equation (12) to simplify the equation (13) for $F$.

$$
F=D\left(\frac{d u_{0}}{d r}+\frac{2}{r} u_{0}\right) \frac{1}{v^{2}} \frac{d^{2} u_{0}}{d t^{2}}+E\left(\frac{d^{2} u_{0}}{d r^{2}} \frac{d u_{0}}{d r}+\frac{1}{r}\left(\frac{d u_{0}}{d r}\right)^{2}-\frac{u_{0}^{2}}{r^{3}}\right)
$$

Two time-dependence cases for the primary field $u_{0}$ will be considered: monochromatic and the impulse like. In the next section, this method will be applied to the problem of propagating monochromatic elastic spherical waves in an isotropic homogeneous nonlinear medium.

\section{Monochromatic Primary Wave}

We seek the solution for the primary wave

$$
u_{0}=I \frac{\sin (\omega t-z)+z \cos (\omega t-z)}{r z}, \quad z=\frac{\omega r}{v}
$$

where $I$ is an arbitrary constant. After substitution of (18) in (17), function $F$ has the form

$$
F=F_{0}(r)+F_{s}(r) \sin 2(\omega t-z)+F_{c}(r) \cos 2(\omega t-z)
$$

where functions $F_{0}(r), F_{s}(r), F_{c}(r)$ are finite sums of negative powers of $r$

$$
\begin{gathered}
F_{0}(r)=-I^{2} D \frac{k^{2}}{2 r^{3}}-I^{2} E \frac{k^{3}}{2 r^{2}}\left(\frac{1}{z^{3}}+\frac{9}{z^{5}}\right) \\
F_{c}(r)=I^{2} \frac{k^{2}}{r^{3}}\left[\frac{D}{2}+E\left(2-\frac{17}{2 z^{2}}+\frac{9}{2 z^{4}}\right)\right]
\end{gathered}
$$




$$
F_{s}(r)=I^{2} \frac{k^{3}}{r^{2}}\left[-\frac{D}{2}+E\left(-\frac{1}{2}+\frac{5}{z^{2}}-\frac{9}{z^{4}}\right)\right]
$$

The structure of (19) allows us to seek the solution for $u_{1}$ in the form

$$
u_{1}(r, t)=u_{1}^{0}(r)+\operatorname{Re}\left(e^{2 i \omega t} \tilde{u}_{1}(r)\right)
$$

where function $u_{1}^{0}(r)$ satisfies the equation

$$
\frac{d^{2} u_{1}^{0}}{d r^{2}}+\frac{2}{r} \frac{d u_{1}^{0}}{d r}-\frac{2}{r^{2}} u_{1}^{0}=-\frac{F_{0}}{\lambda+2 \mu}
$$

and for the complex function $\tilde{u}_{1}(r)$, we have inhomogeneous spherical Bessel equation:

$$
\frac{d^{2} \tilde{u}_{1}}{d r^{2}}+\frac{2}{r} \frac{d \tilde{u}_{1}}{d r}+\left(4 k^{2}-\frac{2}{r^{2}}\right) \tilde{u}_{1}=-\frac{F_{c}-i F_{s}}{\lambda+2 \mu} e^{-2 i z}, \quad k=\frac{\omega}{v}
$$

Solutions for equation (25) when its right-hand side has the form $\frac{e^{-i k r}}{r^{n}}, n=2,3,4,5,6,7$ can be found in Appendix A., which after some algebra enable us to obtain the expression for the nonlinear field:

$$
u_{1}=-\frac{I^{2} D k^{3}}{\lambda+2 \mu}\left(\frac{1}{4 z}+u_{1}^{(1)}(z)\right)-\frac{I^{2} E k^{3}}{\lambda+2 \mu}\left(-\frac{1}{8 z^{3}}-\frac{1}{4 z^{5}}+u_{1}^{(2)}(z)\right)
$$

where

$$
\begin{gathered}
u_{1}^{(1)}(z)=\frac{1}{4} \operatorname{Re}\left(e^{2 i \omega t}\left[h_{1}^{(1)}(2 z) E i(-i 4 z)+h_{1}^{(2)}(2 z) \ln 2 z\right]\right) \\
u_{1}^{(2)}(z)=\frac{1}{4} \operatorname{Re}\left(e^{2 i \omega t}\left[h_{1}^{(1)}(2 z) E i(-i 4 z)+h_{1}^{(2)}(2 z) \ln 2 z+\left(-\frac{3 i}{2}-\frac{5}{2 z}+\frac{2 i}{z^{2}}+\frac{1}{z^{3}}\right) \frac{e^{-2 i z}}{z^{2}}\right]\right)
\end{gathered}
$$

with first index spherical Hankel functions

$$
h_{1}^{(1)}(z)=-\frac{i+z}{z^{2}} e^{i z}, \quad h_{1}^{(2)}(z)=\frac{i-z}{z^{2}} e^{-i z}
$$

of the first and second kind, respectively. Function $E i(-i x)$ is defined by

$$
E i(-i x)=-\int_{x}^{\infty} \frac{e^{-i \xi}}{x} i d \xi
$$


Any function of the form

$$
u_{1}^{(0)}=\frac{a_{0}}{z^{2}}+a_{1} \frac{\sin (\omega t-z)+z \cos (\omega t-z)}{r z}+a_{2} \frac{\cos (\omega t-z)-z \sin (\omega t-z)}{r z}
$$

with arbitrary coefficients $a_{0}, a_{1}, a_{2}$ may be added to the solution (26) to match boundary conditions at reference radius $R$. In the far field, when $z>>1$, the nonlinear field (26) has the asymptotic value

$$
u_{1}=I^{2} \frac{\omega^{2}}{v^{2}} \frac{(3 \lambda+6 \mu+2 A+6 B+2 C)}{8(\lambda+2 \mu)} \frac{\ln (r / R)}{r} \cos 2(\omega t-z)
$$

Assuming that $x=r-R<<R$, we obtain from (32):

$$
u_{1}=I^{2} \frac{\omega^{2}}{v^{2}} \frac{(3 \lambda+6 \mu+2 A+6 B+2 C)}{8(\lambda+2 \mu)} \frac{x}{R^{2}} \cos 2(\omega t-z)
$$

which differs from the result for the plane compressional primary wave (Polyakova, 1964) by a factor of $\frac{1}{R^{2}}$.

The obtained analytical solutions of equation (24) are verified by comparison with its finite-difference solutions.

\section{$5 \quad$ Impulse Primary Wave}

Here we consider the nonlinear propagation of the pulse generated by the primary wave

$$
u_{0}=\frac{q_{, \tau}(\tau)}{v r}+\frac{q(\tau)}{r^{2}}, \quad \tau=t-\frac{r}{v}
$$

where source function $q(\tau)$ describes an initial waveform. Substituting (34) in (17) and taking the inverse Fourier transform over $t$ we have

$$
\begin{gathered}
f(\omega)=\frac{1}{\sqrt{2 p i}} \int_{-\infty}^{\infty} F(t) e^{-i \omega t} d t=-\frac{D}{\lambda+2 \mu} \frac{Q_{2}}{v^{4} r^{3}} e^{-i k r}\left(1+\frac{i k r}{2}\right) \\
-\frac{E}{\lambda+2 \mu}\left[\frac{i k}{2 v^{4} r^{2}} Q_{2}+\frac{1}{v^{2}}\left(-\frac{k^{2}}{r^{3}}+\frac{i k}{r^{4}}+\frac{1}{r^{5}}\right) Q_{1}+\left(-\frac{i k^{3}}{r^{4}}-\frac{4 k^{2}}{r^{5}}+\frac{9 i k}{r^{6}}+\frac{9}{r^{7}}\right) Q_{0}\right] e^{-i k r}
\end{gathered}
$$


Functions $Q_{0}, Q_{1}$, and $Q_{2}$ from (35) depend on waveform $q(\tau)$ of the primary signal:

$$
\begin{aligned}
& Q_{0}=\frac{1}{\sqrt{2 \pi}} \int_{-\infty}^{\infty} q^{2}(\tau) e^{-i \omega \tau} d \tau \\
& Q_{1}=\frac{1}{\sqrt{2 \pi}} \int_{-\infty}^{\infty} q_{, \tau}^{2}(\tau) e^{-i \omega \tau} d \tau \\
& Q_{2}=\frac{1}{\sqrt{2 \pi}} \int_{-\infty}^{\infty} q_{, \tau \tau}^{2}(\tau) e^{-i \omega \tau} d \tau
\end{aligned}
$$

Therefore, in a frequency domain, a nonlinear component satisfies the equation

$$
\frac{d^{2} \tilde{u}_{1}}{d r^{2}}+\frac{2}{r} \frac{d \tilde{u}_{1}}{d r}+\left(k^{2}-\frac{2}{r^{2}}\right) \tilde{u}_{1}=f
$$

which is similar to equation (25). Integrating (39) and returning to the time domain, we have for the nonlinear field

$$
\begin{gathered}
u_{1}=-\frac{D}{\sqrt{2 \pi}(\lambda+2 \mu)} \frac{1}{4 v^{4}} \int_{-\infty}^{\infty} k g_{1}(k r) Q_{2} e^{i \omega t} d \omega \\
-\frac{E}{\sqrt{2 \pi}(\lambda+2 \mu)} \int_{-\infty}^{\infty}\left(\frac{k}{4 v^{4}} g_{2}(k r) Q_{2}+\frac{k^{3}}{v^{2}} g_{3}(k r) Q_{1}+k^{5} g_{4}(k r) Q_{0}\right) e^{i \omega t} d \omega
\end{gathered}
$$

with dimensionless functions $g_{i}(x)$ that have forms

$$
\begin{gathered}
g_{1}(x)=h_{1}^{(1)}(x) E i(-i 2 x)+h_{1}^{(2)}(x) \ln x \\
g_{2}(x)=h_{1}^{(1)}(x) E i(-i 2 x)+h_{1}^{(2)}(x) \ln x+\frac{2 i}{x^{2}} e^{-i x} \\
g_{3}(x)=\frac{1+2 i x}{4 x^{3}} e^{-i x} \\
g_{4}(x)=-\frac{e^{-i x}}{2 x^{3}}\left(\frac{1}{2}-\frac{i}{x}-\frac{1}{x^{2}}\right)
\end{gathered}
$$


Integrating in(40), we obtain

$$
u_{1}=-\frac{D+E}{\lambda+2 \mu} I_{1}(r)+\frac{E}{\lambda+2 \mu} I_{2}(r)
$$

where

$$
\begin{gathered}
I_{1}(r)=\frac{1}{4 r v^{4}}\left(q_{, t t}^{2}\left(t_{0}\right) \ln r+\int_{r}^{\infty} q_{, t t}^{2}\left(t+\frac{r}{v}-\frac{2 x}{v}\right)\left(\frac{2 \ln x}{r}-\frac{1}{x}\right) d x\right) \\
I_{2}(r)=\frac{2 q, t t\left(t_{0}\right) q\left(t_{0}\right)+3 q_{, t}^{2}\left(t_{0}\right)}{4 v^{2} r^{3}}+\frac{q_{, t t}\left(t_{0}\right) q_{, t}\left(t_{0}\right)}{v^{3} r^{2}} \\
+\frac{q\left(t_{0}\right) q_{, t}\left(t_{0}\right)}{v r^{4}}+\frac{q^{2}\left(t_{0}\right)}{2 r^{5}}-\frac{1}{2 v^{3} r^{2}} \int_{-\infty}^{t_{0}} q_{, t t}^{2}(\tau) d \tau, \quad t_{0}=t-\frac{r}{v}
\end{gathered}
$$

An arbitrary field of the form (34) may be added to solution (45) to match the boundary conditions. This specifically enables us to represent equation (45) in the form

$$
I_{1}(r)=\frac{1}{4 r v^{4}}\left(q_{, t t}^{2}\left(t_{0}\right) \ln \frac{r}{R}+v \int_{-\infty}^{t_{0}} q_{, t t}^{2}(\tau)\left[\frac{1}{r} \ln \left(\frac{v t+r-v \tau}{2 R}\right)-\frac{1}{v t+r-v \tau}\right] d \tau\right)
$$

where the far-field part of the nonlinear field becomes equal to zero at $r=R$. Therefore, in the far-field approximation, we have

$$
u_{1}=-\frac{(3 \lambda+6 \mu+2 A+6 B+2 C)}{4(\lambda+2 \mu) v^{4}} \frac{\ln (r / R)}{r} q_{, t t}^{2}\left(t-\frac{r}{v}\right) .
$$

The obtained forms for nonlinear field make it equal to zero at the reference radius $r=R$.

It is a quite common case when the velocity of the displacement, rather than displacement itself is being recorded. Taking the time derivative of the field (45), we have for the velocity of the total-field displacement

$$
\begin{gathered}
u_{, t}=\frac{q_{, t t}\left(t_{0}\right)}{v r}+\frac{q_{, t}\left(t_{0}\right)}{r^{2}}= \\
-\frac{D+E}{\lambda+2 \mu} \frac{1}{2 r v^{4}}\left(q_{, t t}\left(t_{0}\right) q_{, t t t}\left(t_{0}\right) \ln r+\frac{v}{r}(1-2 \ln r) q_{, t t}^{2}\left(t_{0}\right)+v \int_{r}^{\infty} q_{, t t}^{2}\left(t+\frac{r}{v}-\frac{x}{2 v}\right)\left(\frac{2}{r x}+\frac{1}{x^{2}}\right) d x\right)
\end{gathered}
$$


$-\frac{E}{\lambda+2 \mu}\left(\frac{2 q_{, t t t}\left(t_{0}\right) q_{, t}\left(t_{0}\right)+q_{, t t}^{2}\left(t_{0}\right)}{2 v^{3} r^{2}}+\frac{q_{, t t t}\left(t_{0}\right) q\left(t_{0}\right)+4 q_{, t t}\left(t_{0}\right) q_{, t}\left(t_{0}\right)}{2 v^{2} r^{3}}+\frac{q_{, t t}\left(t_{0}\right) q\left(t_{0}\right)+q_{, t}^{2}\left(t_{0}\right)}{v r^{4}}+\frac{q\left(t_{0}\right) q_{, t}\left(t_{0}\right)}{r^{5}}\right)$

Obtained analytical solutions for equation (39) are verified by comparison with its finite-difference solutions. Expressions for the source function $q_{\tau}$ can be found in Appendix B.

\section{Discussion}

Obtained results for nonlinear field components show a complex dependence of near-field terms on propagation distance. If field measurements are taken close to powerful sources (such as explosions), then this complexity should be taken into account when estimating medium parameters (nonlinear constants and attenuation).

If the condition

$$
\beta_{0}=\frac{D+E}{\lambda+2 \mu}=\frac{3(\lambda+2 \mu)+4 m+2 l}{\lambda+2 \mu}=0
$$

is satisfied, then the nonlinear field vanishes in the far field. Using data from Huges and Kelly (1953) for solid materials, we have $\beta_{0}=-6.5$ for polystyrene, $\beta_{0}=-7.3$ for armco iron, $\beta_{0}=4.4$ for pyrex. Observations in rock showed much higher nonlinear coefficients. For sandstone, $\beta_{0}$ can be as high as 7000 (Johnson et al.,1993). These evaluations, however, were obtained for small strains on the order of $10^{-5}-10^{-4}$. Seismic waves caused by large explosions and earthquakes strains have the order of $10^{-3}-10^{-2}$.

\section{Conclusions}

Solutions have been presented for spherical wave propagation in cases involving the harmonical time dependence of a primary wave and a pulse. It was shown that a nonlinear component in the far field has resonant character and $\ln r / r$ dependence as propagation distance $r$ grows. A nonlinear field gives relative enhancement to both low-frequency and high-frequency parts of the total field. The near-field component of the primary field does not generate a far field of the nonlinear component (in the approximation considered). A numerical approach is required to reveal the details of the nonlinear field generation. 


\section{Acknowledgments}

This work was supported by the U.S. Department of Energy under Contract No. DE-ACO2-05CH11321. Discussions with Lane Johnson helped to improve the paper.

\section{Bibliorgaphy}

Banghar, A.R., References Source time function and spectra for underground explosions, in: Journal of Earth System Science, 92, DOI 10.1007/BF02866740, 1983.

Beresnev,I., Numerical model of the spherical elastic wave propagation in a nonlinear medium, in: Int.J. of Modern Physics C,2(1),pp. 250-253, 1990.

Daley T.M., Peterson J.E. and McEvilly T.M., A search for evidence of nonlinear elasticity in the earth, - Science report, DOE, LBL-33313, UC-403, 1992.

Gedroits A.A. and Krasil'nikov V.A., Finite-amplitude elastic waves amplitude in solids and deviations from the Hook's law., in: Zh.Eksp. Teor.Fiz. (Sov. Phys.-JETP), 16,pp. 1122-1130, 81-97, 1963.

Gol'dberg Z.A., Interaction of plane longitudinal and transverse elastic waves,Soviet Phys.- Acoust., Vol.6,N3, pp. 306-310, 1961.

Johnson P.A., T.J. Shankland, R.G. O'Connel, and J.N. Albright, Nonlinear generation of elastic waves in crystalline rock,J.G.R., Vol.92, pp. 3597-3602, 1987.

Hughes D.S. and Kelly J.L., Second-order elastic deformations in solidsPhys.Rev., Vol.92,N5,,pp. 1145$1149,1953$.

Jones G.L. and Kobett D.R., Interaction of elastic waves in an isotropic solid, J.Acoust.Soc.of Am.,, Vol.35,N1,pp.5-10, 1963.

Korneev, V. A., K.T.Nihei, and L.R. Myer, Nonlinear interaction of plane elastic waves, in: Science report, DOE, LBNL-41914. 1998.

Korneev, V. A., Time domain solutions for nonlinear elastic 1-D plane wave propagation, in: Science report, DOE, LBNL-37411. 1998.

Landau L.D. and Lifshitz E.M., Theory of elasticity. New York, John Wiley.,1953.

Murnaghan F.D., Finite deformation of elastic solid. New York, John Wiley.,1951.

Polyakova A.L., Nonlinear effects in a solid, Sov.Phys. - Solid state,, Vol.6,N1, 1964. 
Saikia, C. K.; Helmberger, D. V.; Stead, R. J.; Woods, B. B., Effects of Source RDP Models and Nearsource Propagation: Implication for Seismic Yield Estimation, Pure and Applied Geophysics, Volume 158, Issue 11, pp. 2173-2216, 2001.

Taylor L.H. and Rollins F.R., Ultrasonic study of three-phonon interactions. I. Theory , , Phys. Rew., Vol.136, 3A, A591-A596. , 1999.

Zarembo L.K. and Krasil'nikov V.A., Nonlinear phenomena in the propagation of elastic waves in solids, in: Soviet Physics Uspekhi,Vol.13,N6, pp.778-797,1971.

Zarembo L.K. and Krasil'nikov V.A., Introduction in nonlinear acoustics, in: Moscow,Science,1966.

Zhukov, A. P., K. I. Loginov, M. B. Shneerson, V. E. Shulakova, R. G. Kharisov and V. A. Ekimenko, Nonlinear properties of vibrator-generated wavefields and their application to hydrocarbon detection, in: The Leading Edge, v. 26; no. 11; p. 1395-1402; DOI: 10.1190/1.2805768, 2007

\section{Appendix A}

The following equation

$$
\frac{d^{2} u}{d r^{2}}+\frac{2}{r} \frac{d u}{d r}+\left(k^{2}-\frac{2}{r^{2}}\right) u=\frac{e^{-i z}}{r^{n}}, \quad z=k r
$$

has the following solutions:

$$
\begin{array}{rlrl}
u & =-\frac{i}{2}\left(h_{1}^{(1)}(z) E i(-i 2 z)+h_{1}^{(2)}(z) \ln z+\frac{2 i}{z^{2}} e^{-i z}\right), & & \text { for } n=2 \\
u & =-\frac{i k}{2} \frac{e^{-i z}}{z^{2}}, & & \text { for } n=3 \\
u & =-\frac{i k^{2}}{6}\left(2 h_{1}^{(1)}(z) E i(-i 2 z)+\frac{i}{z^{2}} e^{-i z}\right), & \text { for } n=4 \\
u=\frac{i k^{3}}{2}\left(\frac{2 i}{3} h_{1}^{(1)}(z) E i(-i 2 z)-\left(\frac{i}{2}+\frac{z}{3}\right) \frac{e^{-i z}}{z^{3}}\right), & \text { for } n=5 \\
u=\frac{i k^{4}}{10}\left(2 h_{1}^{(1)}(z) E i(-i 2 z)+\left(i z-\frac{3}{2}-\frac{i}{z}\right) \frac{e^{-i z}}{z^{3}}\right), & \text { for } n=6 \\
u=\frac{i k^{5}}{18}\left(-\frac{i 8}{5} h_{1}^{(1)}(z) E i(-i 2 z)+\left(\frac{4 z}{5}+\frac{6 i}{5}-\frac{4}{5 z}-\frac{i}{z^{2}}\right) \frac{e^{-i z}}{z^{3}}\right), & \text { for } n=7
\end{array}
$$

with first index spherical Hankel functions

$$
h_{1}^{(1)}(x)=-\frac{i+x}{x^{2}} e^{i x}, \quad h_{1}^{(2)}(x)=\frac{x-i}{x^{2}} e^{-i x}
$$


of the first and second kind, respectively. Function $E i(-i x)$ is defined by

$$
E i(-i x)=-\int_{x}^{\infty} \frac{e^{-i \xi}}{\xi} d \xi
$$

\section{Appendix B}

For a step pressure source when the pressure inside of a spherical cavity has the form

$$
P(t)=P_{0} U(t),
$$

where $U(t)$ is a unit step (Heavyside) function, we have the following expressions for $q(\tau)$ and its derivatives

$$
\begin{aligned}
q(\tau) & =U(\tau) \frac{P_{0} R^{3}}{4 \mu}\left(1-e^{-\eta \frac{v}{R} \tau}\left[\cos \left(\omega_{0} \tau\right)+\frac{\eta}{\sqrt{2 \eta-\eta^{2}}} \sin \left(\omega_{0} \tau\right)\right]\right) \\
q_{, \tau}(\tau) & =U(\tau) \frac{P_{0} R^{2} v}{2 \mu} \frac{\eta}{\sqrt{2 \eta-\eta^{2}}} e^{-\eta \frac{v}{R} \tau} \sin \left(\omega_{0} \tau\right) \\
q_{, \tau \tau}(\tau) & =U(\tau) \frac{P_{0} R v^{2} \eta}{2 \mu} e^{-\eta \frac{v}{R} \tau}\left[\cos \left(\omega_{0} \tau\right)-\frac{\eta}{\sqrt{2 \eta-\eta^{2}}} \sin \left(\omega_{0} \tau\right)\right] \\
q_{, \tau \tau \tau}(\tau) & =U(\tau) \frac{P_{0} v^{3} \eta^{2}}{\mu} e^{-\eta \frac{v}{R} \tau}\left[\frac{\eta-1}{\sqrt{2 \eta-\eta^{2}}} \sin \left(\omega_{0} \tau\right)-\cos \left(\omega_{0} \tau\right)\right]
\end{aligned}
$$

Waveforms for Haskell's reduced displacement potential (Banghar,1983; Saikia et al., 2001) are described by the functions

$$
\begin{aligned}
q(\tau) & =U(\tau)\left[1-e^{-k \tau}\left(1+k \tau+\frac{(k \tau)^{2}}{2}+\frac{(k \tau)^{3}}{6}-B_{0}(k \tau)^{4}\right)\right] \\
q_{, \tau}(\tau) & =U(\tau) k e^{-k \tau}\left(\left(4 B_{0}+\frac{1}{6}\right)(k \tau)^{3}-B_{0}(k \tau)^{4}\right) \\
q_{, \tau \tau}(\tau) & =U(\tau) k^{2} e^{-k \tau}\left(\left(12 B_{0}+\frac{1}{2}\right)(k \tau)^{2}-\left(8 B_{0}+\frac{1}{6}\right)(k \tau)^{3}+B_{0}(k \tau)^{4}\right) \\
q_{, \tau \tau \tau}(\tau) & =U(\tau) k^{3} e^{-k \tau}\left(\left(24 B_{0}+1\right) k \tau-\left(36 B_{0}+1\right)(k \tau)^{2}+\left(12 B_{0}+\frac{1}{6}\right)(k \tau)^{3}-B_{0}(k \tau)^{4}\right)
\end{aligned}
$$

where

$$
k R=\frac{24 B_{0}+1}{6 B_{0}}
$$

and constant $B_{0}$ has a value in the $0.2-0.8$ range. 